\title{
Optofluidic Sensor for Inline Hemolysis Detection on Whole Blood
}

Zhou, Chen; Keshavarz Hedayati, Mehdi; Zhu, Xiaolong; Nielsen, Frank; Levy, Uriel; Kristensen, Anders

Published in:

ACS Sensors

Link to article, DOI:

10.1021/acssensors.8b00030

Publication date:

2018

Document Version

Publisher's PDF, also known as Version of record

Link back to DTU Orbit

Citation (APA):

Zhou, C., Keshavarz Hedayati, M., Zhu, X., Nielsen, F., Levy, U., \& Kristensen, A. (2018). Optofluidic Sensor for Inline Hemolysis Detection on Whole Blood. ACS Sensors, 3(4), 784-791.

https://doi.org/10.1021/acssensors.8b00030

\section{General rights}

Copyright and moral rights for the publications made accessible in the public portal are retained by the authors and/or other copyright owners and it is a condition of accessing publications that users recognise and abide by the legal requirements associated with these rights.

- Users may download and print one copy of any publication from the public portal for the purpose of private study or research.

- You may not further distribute the material or use it for any profit-making activity or commercial gain

- You may freely distribute the URL identifying the publication in the public portal

If you believe that this document breaches copyright please contact us providing details, and we will remove access to the work immediately and investigate your claim 


\title{
Optofluidic Sensor for Inline Hemolysis Detection on Whole Blood
}

\author{
Chen Zhou, ${ }^{\dagger}$ Mehdi Keshavarz Hedayati, ${ }^{\dagger}$ Xiaolong Zhu, ${ }^{\dagger}$ Frank Nielsen, ${ }^{\ddagger}$ Uriel Levy, ${ }^{\text {II }}$ \\ and Anders Kristensen* ${ }^{\dagger} \dagger$ (1)
†Department of Micro- and Nanotechnology, Technical University of Denmark, DK-2800 Kongens Lyngby, Denmark
${ }^{*}$ Radiometer Medical ApS. DK-2700 Brønshøj, Denmark

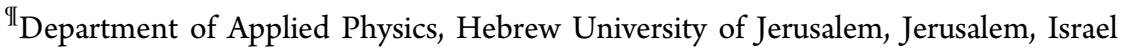

\section{Supporting Information}

ABSTRACT: Hemolysis is the rupture of red blood cells and constitutes the most common reason for unsuitable blood samples in the clinic. To detect hemolysis, one has to separate the hemoglobin in blood plasma from that in red blood cells. However, current methods entail centrifugation for cell-plasma separation, which is complex, time-consuming, and not easy to integrate into point-of-care $(\mathrm{PoC})$ systems. Here, we demonstrate an optofluidic sensor composed of nanofilters on an optical waveguide, which enables evanescent-wave absorption measurement of hemoglobin in plasma with the capability of real-time inline detection on whole blood without extra sample preparation like centrifugation. Long-term testing with inline integration in a modified, commercial blood gas analyzer shows high reliability and repeatability of the measurements even with the presence of interference from bilirubin. We envision that the present work has large potential in improving diagnosis quality by enabling $\mathrm{PoC}$ hemolysis detection in blood gas analyzers and can also lend unique sensing capabilities to other applications dealing with complex turbid media.

KEYWORDS: hemolysis sensor, Point-of-Care, whole blood, optofluidics, nanowells, waveguide sensor, nanoimprint, in vitro diagnostics

$\mathrm{H}$ emolysis is the rupture of the red blood cells (RBCs) and the release of their contents into blood plasma. ${ }^{1}$ It is the largest preanalytical error source in health care facilities and clinical laboratories around the world, accounting for 39-69\% of all the unsuitable samples-about five times higher than the second leading cause. ${ }^{2}$ Hemolysis can originate from both in vivo conditions, such as sickle cell disease and infections, as well as in vitro conditions, such as traumatic blood draw and sample mishandling; the possibility of both occurring complicates identification of the source of hemolysis. Furthermore, the presence of hemolysis interferes with the detection of important vital parameters like $\mathrm{K}^{+}$and $\mathrm{Ca}^{+}$concentration in the blood. ${ }^{1}$ In other situations like cardiac surgery undergoing cardiopulmonary bypass (CPB), hemolysis is nearly ubiquitous, ${ }^{3}$ eventually causing acute kidney injury. ${ }^{4}$ The state-of-the art hemolysis detection protocols require centrifugation steps to separate blood plasma and cells, which is time-consuming, resource demanding, and prone to cause additional preanalytical errors. Centrifugation protocols are not easy to integrate into PoC platforms.

In this paper, we present a $\mathrm{PoC}$ hemolysis sensor combining evanescent absorption detection and local plasma separation without the need of any sample preparation. The plasma separation is achieved with an integrated nanofilter comprising an array of nanowells. So far, waveguide absorption spectroscopy has been demonstrated for sensing $\mathrm{pH}$ value, protein, $^{6-9}$ and molecules. ${ }^{10-15}$ Separation of blood plasma and RBCs is achieved by either active methods, such as magnetic, ${ }^{16,17}$ dielectrophoretic, ${ }^{18,19}$ and acoustic, ${ }^{20,21}$ or passive methods, such as hydrodynamic, ${ }^{22-24}$ sedimentation, 25,26 and filtration. ${ }^{27-30}$ Our optofluidic strategy is based on an innovative dual function of nanofilters that act as a component of the waveguide as well as a functional filter for RBCs. The nanofilters enable light propagation without diffraction and scattering while effectively blocking cells or platelets with short diffusion length and time, as shown schematically in Figure 1a. Grating couplers are integrated to couple broadband LED light into-and spectrally disperse light out of-the waveguide sensor, enabling spectral analysis without using an extra spectrometer or monochromator. The wavelength range of the LED light source is adjusted to the highest optical absorption of $\mathrm{Hb}$, as shown in Figure $1 \mathrm{~b}$ (highlighted area). The absorbance measured with the optofluidic sensor is compared with photospectrometer reference data. The sensor was integrated into a modified, commercial blood gas analyzer (ABL90 FLEX, Radiometer Medical ApS, Denmark), as shown in Figure 1c, to assess its repeatability, reliability, and durability.

\section{RESULTS AND DISCUSSION}

Sensor Design and Optical Simulation. The sensor is designed to have dual-function: a waveguide and a filter for RBCs. The waveguide is made by the assembly of a cladding

Received: January 10, 2018

Accepted: February 21, 2018 

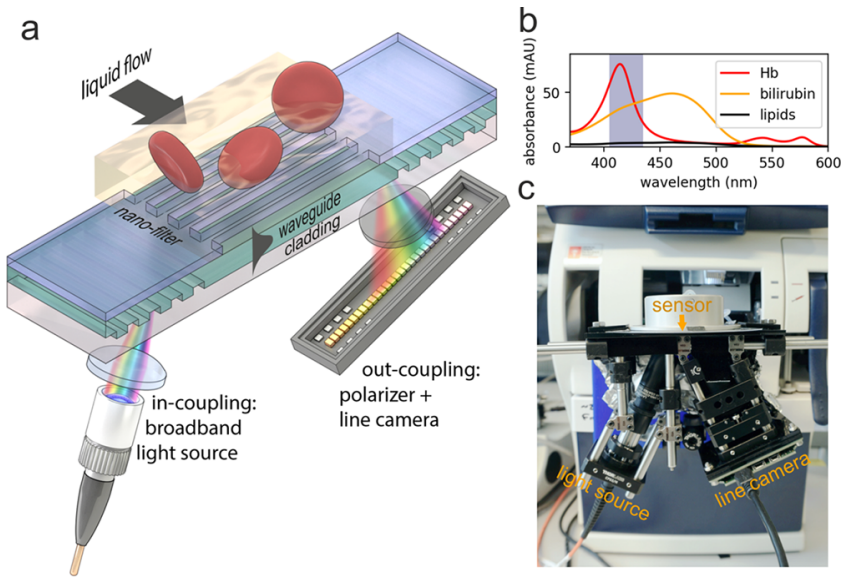

Figure 1. (a) Illustration of the hemolysis sensor comprising waveguide, cladding, and nanofilter for cell/plasma separation. Gratings functioning as dispersive element enable spectral analysis with a line camera as readout. (b) Reference absorption spectra of main components in blood plasma: $\mathrm{Hb}$, bilirubin, and lipids. The wavelength of LED light used is highlighted at the background. (c) Portable setup attached to a state-of-the-art blood gas analyzer for automatized measurements.

layer, a waveguide core layer, and a structured top layer for filtering. To effectively and reliably eliminate the absorption from the RBCs (smallest feature size of about $1-2 \mu \mathrm{m}$ ), these nanofilters have a pitch width of $400 \mathrm{~nm}$ and an opening of 200 $\mathrm{nm}$ for blood plasma/light interaction. Only species smaller than the size of the filter opening are allowed to diffuse downward and thus interact with the guided light, as illustrated in Figure 2a. The small dimension prevents cells clogging the filter. The nanofilters are aligned parallel to the light propagation direction without interruption to enable the wave propagation without diffraction and scattering. The cladding layer on top of grating couplers is not structured in order to prevent coupling conditions being influenced by changes of refractive index (RI) in the liquid sample. According to Lambert-Beer's law, we determined the sensitivity $S$ in our system with relation to other parameters of the sensor as follows (derivation in Supporting Information)

$$
S=\eta \varepsilon l
$$

where $\eta$ denotes the mode-field overlap with the analyte. ${ }^{31} \eta$ is defined as the energy ratio of the evanescent field interacting with the liquid to the total energy flow. $\varepsilon$ is the extinction coefficient of the detection target; $l$ the length of the light/ sample interaction. If the length of the sensor is fixed by a given specification, the mode-field overlap becomes the major optimization parameter. Mode analysis was performed using COMSOL Multiphysics to optimize the mode-field overlap and thus the sensitivity (rf. Materials and Methods). Mode analysis of the fabricated ridge waveguide is shown in Figure $2 b$, where transverse electric (TE) and transverse magnetic (TM) modes indicate horizontal and vertical electrical field distribution, respectively. In this configuration, the TM mode shows higher mode-field overlap than the TE mode, as Figure 2b (right panel) depicts. The height of the filter and the thickness of the waveguide were swept in simulation for sensitivity optimization. In Figure 2c, the mode-field overlap ratio of the TE mode is plotted. With decreasing waveguide thickness, the overlap ratio increases because of less confinement. On the other hand, with larger thickness, the waveguide supports the guiding of higher-
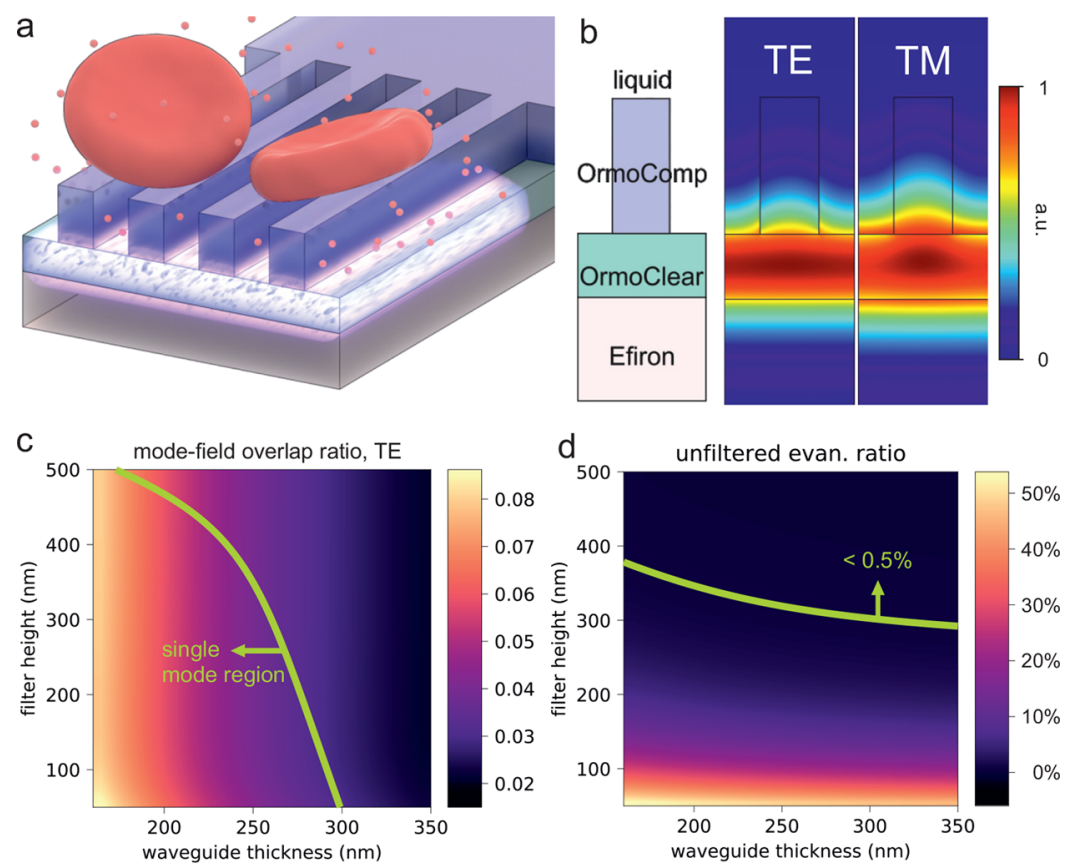

Figure 2. (a) Illustration of the working principle of the waveguide sensor with nanofilters. Large components in blood like cells and platelets are blocked out of the evanescent field of the waveguide. Small molecules like $\mathrm{Hb}$ are measured with evanescent absorption spectroscopically. (b) Mode analysis simulation (electrical field norm) of the fabricated waveguide. Different waveguide layers are illustrated on the left; TE and TM polarizations are shown with their field distribution on the right. (c) Evanescent overlap ratio with TE polarization for varying filter height (OrmoComp) and waveguide thickness (OrmoClear), revealing different sensitivities. The desired region with single mode operation is highlighted. (d) Ratio of the evanescent energy distributed outside the filter range is plotted agaisnt filter height (OrmoComp) and waveguide thickness (OrmoClear). The desired region with $<0.5 \%$ is highlighted. 

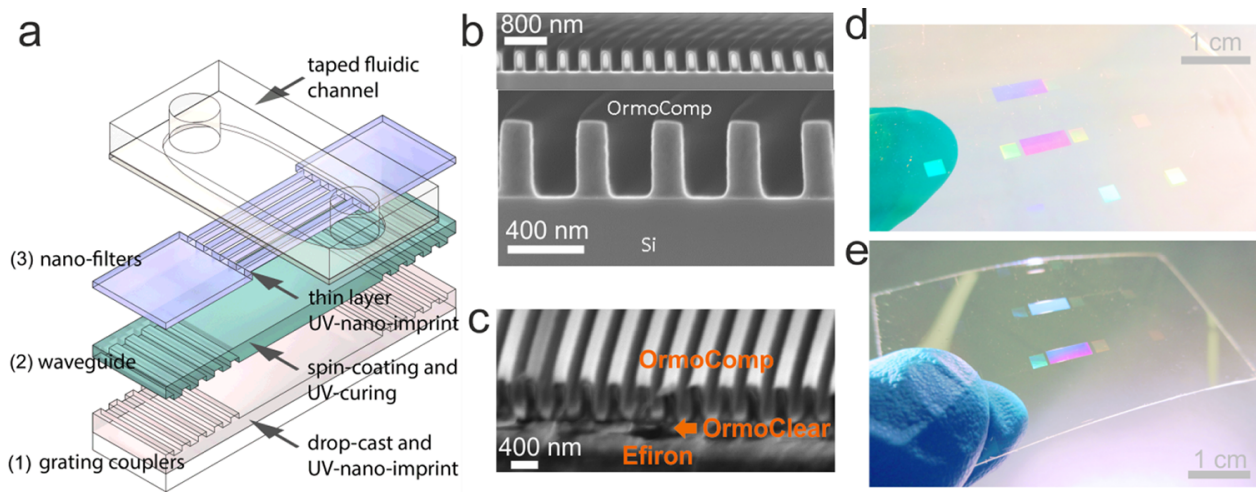

Figure 3. (a) Illustration of the fabrication steps of the sensor utilizing multiple UV-nanoimprint steps. Fluidic channel is easily defined with taped channels. (b) Cross-sectional SEM images of nanofilters fabricated on Si in which the filter height and width can be seen on the bottom panel. The high uniformity is shown on the top panel. (c) Cross-sectional SEM images of the fabricated waveguide with OrmoComp as filters, OrmoClear as waveguide, and Efiron as cladding. (d) Fabricated sensor on a glass wafer. (e) Fabricated sensor on a free-standing membrane.

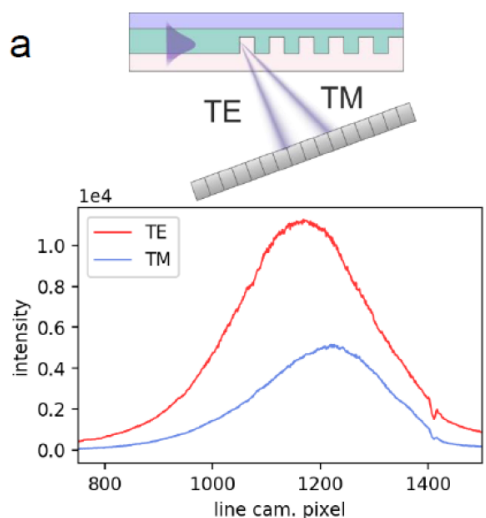

C

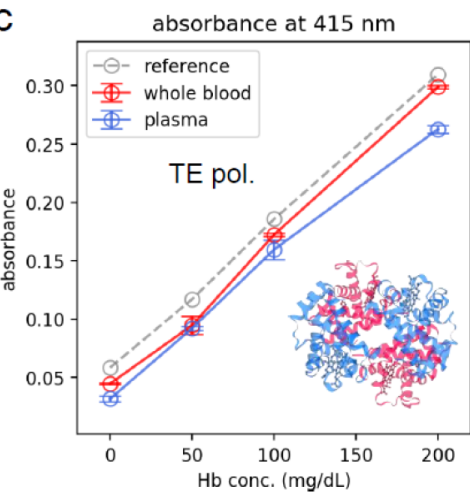

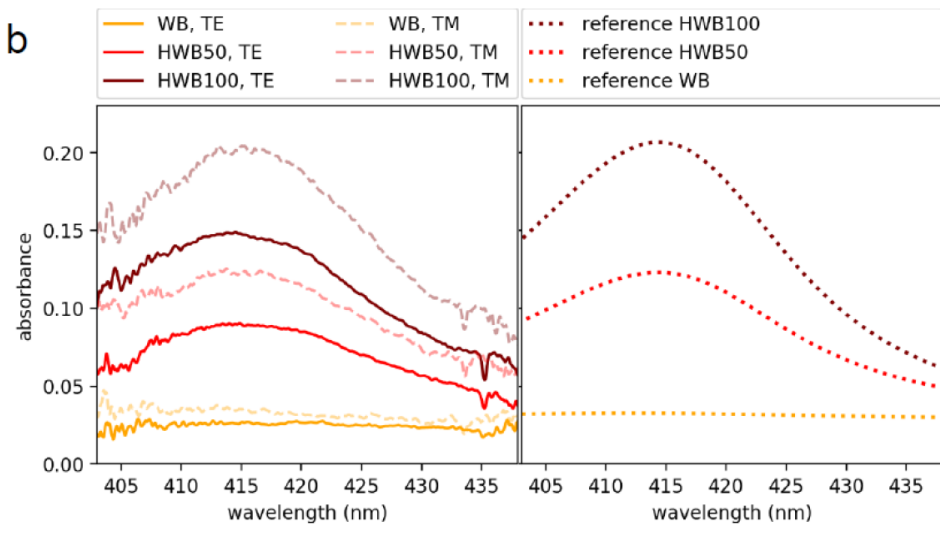

d

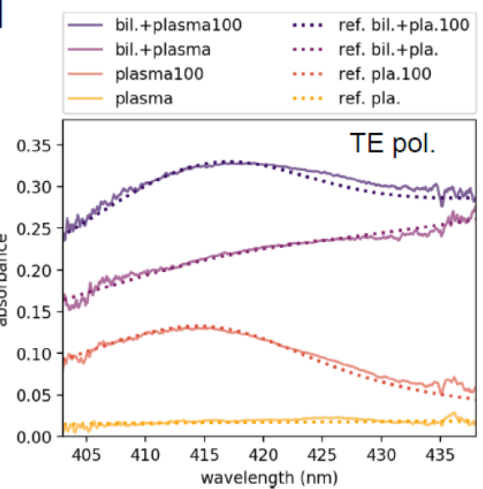

Figure 4. (a) Projection of TE and TM mode on the line camera. (b) Absorption spectra measured with whole blood (WB) and hemolyzed whole blood (HWB, 50 and $100 \mathrm{mg} / \mathrm{dL}$ ) samples with TE and TM modes (left). The photospectrometer reference measurements taken with $1 \mathrm{~cm}$ cuvettes are show on the right. (c) Measurements of different $\mathrm{Hb}$ concentrations in whole blood and plasma. The photospectrometer reference spectra are scaled and plotted at the background. (d) Absorption spectra of plasma, plasma with $100 \mathrm{mg} / \mathrm{dL} \mathrm{Hb}$ and their bilirubin-interfered (20 mg/dL) counterparts. (e) Absorption measured with different concentrations of bilirubin mixed into nonhemolyzed plasma.

order modes. The border where the first order mode occurs is marked in Figure 2c. The higher order modes were excluded for their leaky behavior which would compromise data analysis.

The height of the filters plays an important role in filtration of the RBCs. The area above the filters can be accessed by RBCs causing nonspecific absorption, and must be minimized. Figure $2 \mathrm{~d}$ shows the unfiltered ratio which is defined as the proportion of the propagating mode energy flow above the filter to the corresponding total energy flow in the liquid. $0.5 \%$ is set to be the threshold, which is derived from the desired $\mathrm{Hb}$ detection limit of $20 \mathrm{mg} / \mathrm{dL}$ and the $\mathrm{Hb}$ concentration in $\mathrm{RBCs}$ of about $3600 \mathrm{mg} / \mathrm{dL}$. The dividing line shown in Figure $2 \mathrm{~d}$ marks the $0.5 \%$ limit. The waveguide thickness of $180 \mathrm{~nm}$ and the filter height of $400 \mathrm{~nm}$ were chosen to compromise high sensitivity and reliable filtration. Our fabricated device (filter height $420 \mathrm{~nm}$, filter width $180 \mathrm{~nm}$, waveguide thickness 190 $\mathrm{nm}$ ) fairly matches the designed values from the simulation, in which case the mode-field overlap ratio, $\eta$ is $7.0 \%$ (TM) and $6.0 \%$ (TE), and a unfiltered ratio is lower than $0.5 \%$.

The grating couplers have a periodicity of $368 \mathrm{~nm}$ to enable the coupling of broadband light with wavelengths between 405 and $435 \mathrm{~nm}$ into the waveguide at an incidence angle of 20- 
$28^{\circ}$. There is no lateral confinement in the waveguide, whereas a width of $2 \mathrm{~mm}$ is designed for the grating couplers and the filters. The large dimension is chosen deliberately in order to use commercial LEDs as a light source, which provides broadband illumination and a large focal spot size. Here, the broadband light is coupled with different incident angles. For a $30 \mathrm{~nm}$ bandwidth centered at $420 \mathrm{~nm}$, an incident angle range of $5.8^{\circ}$ is needed to couple the light in, leading to a very low numerical aperture (i.e., 0.05). The focus is strongly limited because of the high beam divergence of the commercial available LED light source. To harvest the maximal energy of the light source, the angles are matched for coupling with a single convex lens and the beam size is controlled to be several millimeters. Note that multilayer gratings can increase the broadband coupling efficiency; ${ }^{9}$ however, it leads to higher costs and complexity during fabrication.

Sensor Fabrication and System Integration. The sensor is fabricated in multiple nanoimprint steps that provide a sub$200 \mathrm{~nm}$ resolution with up-scalable production capacity. Figure 3a shows the entire fabrication sequence. At first, a drop-cast polymer (Efiron, Luvantix, South Korea) is imprinted with UVcuring to define the grating couplers. A second layer (OrmoClear H101, micro resist technology GmbH, Germany) is then spin-coated on top to serve as the core of the waveguide. The final layer featuring the nanofilters (OrmoComp, micro resist technology $\mathrm{GmbH}$, Germany) is also fabricated with UVnanoimprint using an organic-inorganic hybrid polymer stamp (OrmoStamp, micro resist technology $\mathrm{GmbH}$, Germany). With the methods developed here, we successfully demonstrated (i) large scale structural homogeneity up to $6 \times 2 \mathrm{~mm}^{2}$, (ii) sub$200 \mathrm{~nm}$ resolution with an aspect ratio (AR) of 2.5 , (iii) very low imprint residual left to the layer underneath, (iv) very low cost per device, and (v) nearly $100 \%$ fabrication yield. The imprint residual-the thin layer of polymer left on the supporting substrate after the imprint ${ }^{32}$ - must be minimized because it directly decreases the sensitivity. This is achieved by introducing an organic-inorganic hybrid polymer mold replicated from an E-beam written Si stamp. Demonstrations of the imprinted nanofilters on a $\mathrm{Si}$ substrate and on a waveguide are shown in Figure $3 b$ and c, respectively, where the imprint residual layer thickness is below $20 \mathrm{~nm}$. The combination of the materials allows the final device to be flexible and detachable, as the sensor can be simply peeled off the glass substrate. The detached, free-standing, and flexible membrane can be cut into individual sensors, as shown in Figure $3 \mathrm{~d}$ and e. This flexibility grants the unique capability of one-step integration into an existing platforms such as a sensor cassette containing other sensors. In comparison with other highly sensitive evanescent sensors, ${ }^{33-38}$ our sensor can achieve very short turnaround time ( $<60 \mathrm{~s})$ with high specificity toward $\mathrm{Hb}$ in blood plasma. It can measure blood samples with a variable hematocrit (e.g., from $0 \%$ to $45 \%$ ) and is insusceptible to any cell clogging. The separation is immediate and invulnerable to varying flow rate caused by peristaltic pumping, which is hard to achieve by any microfluidic separation methods. ${ }^{21-24}$

Sensor Performance. Using the output grating coupler as the dispersive element, different wavelengths can be resolved from a waveguide by a line camera, as demonstrated by Mendes et al. ${ }^{9}$ The use of a single mode waveguide is important because higher order modes can contaminate the spectrum due to the different out-coupling angles of these modes. In this work, we used a linear polarizer to distinguish the TE and TM polarized light because there is around $2^{\circ}$ difference when they are coupled out of the waveguide. As shown in Figure 4a, the peaks in the spectra of these two modes deviate in position on the camera. In the present configuration, both TE and TM modes are excited by the wide-angle in-coupling. The spectrum on the camera is determined with two lasers with different wavelengths (details in Supporting Information). To compare with reference absorption measurements with a photospectrometer, the spectral sensitivities of the waveguide were taken into account to correct the line shapes.

We tested the sensor with whole blood (WB), both human and bovine. The left panel of Figure $4 \mathrm{~b}$ shows the absorption spectra of WB and hemolyzed whole blood (HWB) spiked with 50 and $100 \mathrm{mg} / \mathrm{dL} \mathrm{Hb}$. The concentration of $100 \mathrm{mg} / \mathrm{dL}$ is an important threshold as it causes about $+7 \%$ and $-24 \%$ interference in clinically relevant $\mathrm{K}^{+}$and total bilirubin concentration in the blood, respectively. ${ }^{39}$ These limits are also close to the values of the allowable errors recommended by Clinical Laboratory Improvement Amendments. ${ }^{40}$ As shown in Figure $4 \mathrm{~b}$, the difference between 0,50 , and $100 \mathrm{mg} / \mathrm{dL}$ is highly distinctive in both TE and TM polarized incidences, which confirms that the sensitivity is sufficiently high. The maximum of the absorption is visible at $415 \mathrm{~nm}$, in good agreement with the line shape of the photospectrometer reference measurements shown on the right panel.

Figure $4 \mathrm{c}$ shows the measurements of WB samples (red line) and their plasma counterparts (blue line) as well as the photospectrometer reference measurements with $1 \mathrm{~cm}$ cuvettes (gray dashed line), where the latter is scaled in amplitude for comparison. The maximum $\mathrm{Hb}$ concentration used is $200 \mathrm{mg} /$ $\mathrm{dL}$, which corresponds to about $0.7 \%$ hemolysis-close to the level recommended by European Directorate for the Quality of Medicines (EDQM) for maximum acceptable level of hemolysis in blood products for transfusion. ${ }^{41}$ Each data point is based on three measurements. At 0 and $100 \mathrm{mg} / \mathrm{dL} \mathrm{Hb}$ concentrations, the coefficients of variation $(\mathrm{CoV})$ are $1.65 \%$ and $0.86 \%$, respectively, showing excellent repeatability of the sensor. Although for the clinical use the hemolysis level from 0 to $200 \mathrm{mg} / \mathrm{dL}$ is the most interesting range, the present sensor can perform in larger extent ranging from 0 to $1000 \mathrm{mg} / \mathrm{dL}$. On the other hand, comparing the whole blood and plasma measurements, we observed very good overlaps which shows that filtration is highly effective. The absorbance of the WB samples is slightly higher than that of the plasma, which can be attributed to the unspecific scattering of the light which can be absorbed by RBCs in the bulk.

In field applications, however, whole blood can be more complex than just hemoglobin in free plasma. There, the presence of bilirubin is a main source of interference. We examined the interference by adding high concentration of bilirubin into the hemolyzed and nonhemolyzed plasma samples. $20 \mathrm{mg} / \mathrm{dL}$ (corresponding to $340 \mathrm{mmol} / \mathrm{L}$ ) bilirubin was added, which is a threshold for neonatal hyperbilirubinaemia that can lead to neurological dysfunction. ${ }^{42}$ As Figure $4 \mathrm{~d}$ shows, the bilirubin addition increases the absorption for both the sensor and reference measurements. However, the exact amount of $\mathrm{Hb}$ and bilirubin can be determined by multivariate regression with the reference spectra. The addition of high concentration bilirubin can increase the overall sensitivity by $18 \%$, which is consistent throughout the measurements over long period and thus can be calibrated. The sensitivity shift is caused by two factors: first, the large difference in molecular weight of $\mathrm{Hb}$ and bilirubin, which are 

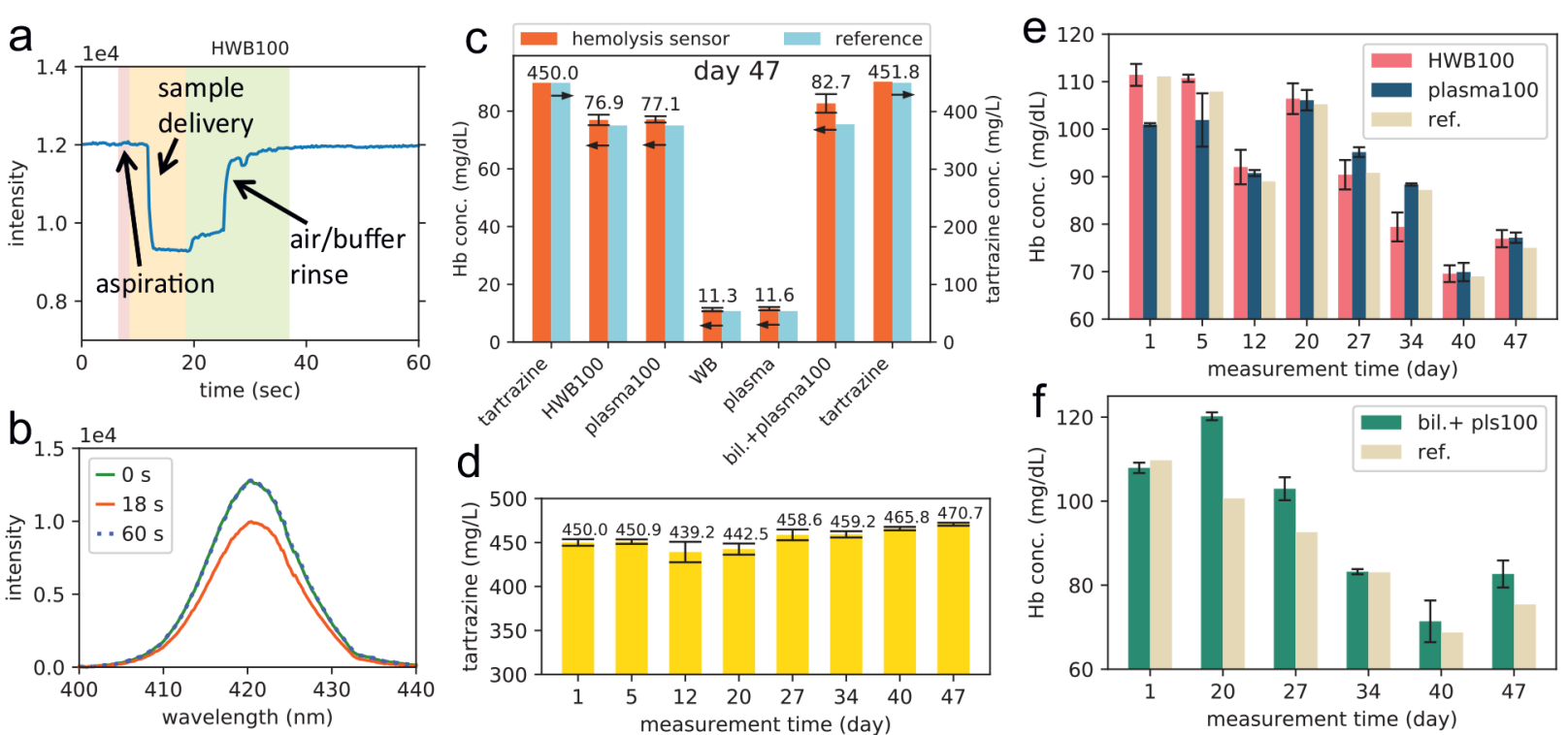

Figure 5. (a) Time-resolved absorption signal at $420 \mathrm{~nm}$ wavelength for measurement of hemolyzed whole blood (HWB) with $100 \mathrm{mg} / \mathrm{dL} \mathrm{Hb}$. Different sections of the measurements are highlighted and annotated. (b) Spectral information during a measurement for buffer signal at 0 and $60 \mathrm{~s}$ and HWB at $18 \mathrm{~s}$. (c) Measurement protocol on 47th measurement day. The values from our sensor are annotated on top and to be compared with references. (d) Sensitivity development with tartrazine calibration liquid for 47 days. (e) Comparison of hemolyzed whole blood ( $\mathrm{Hb} 100 \mathrm{mg} / \mathrm{dL}$, HWB100) and their plasma counterparts (plasma100) together with reference values.(f) Comparison of hemolyzed whole blood (100 mg/dL $\mathrm{Hb}$ ) with bilirubin addition (bil.+pls100) with reference values of the $\mathrm{Hb}$ concentration.

$16 \mathrm{kDa}$ and $0.58-0.76 \mathrm{kDa}$ (unconjugated and conjugated), respectively. The small protein can access the waveguide surface more easily, to which albumin is bound, owing to its high affinity. We observed the same with tartrazine dye solution $(534 \mathrm{~g} / \mathrm{mol})$. Second, $99.9 \%$ of unconjugated bilirubin is bound to albumin in the plasma, ${ }^{43}$ while plasma albumin also adheres to the sensor surface, where the evanescent field is strongest. Different bilirubin concentration mixed into plasma was measured without hemolysis as shown in Figure 4e. Here, we observed no change in the sensitivity in high concentration range, which shows that the change of bilirubin/albumin binding ratio in high concentration is not relevant. ${ }^{44}$ Other nonspecific binding to the sensor surface is not critical for two reasons: (1) The sensor surface is passivated by albumin from the blood plasma. (2) As far as a molecule not absorbing significantly in the $400-430 \mathrm{~nm}$ range, it will not interfere with our absorption measurement, since we analyze the absorption fingerprint of a molecule rather than the refractive index change. In summary, we showed the capability of detecting $\mathrm{Hb}$ on whole blood and distinguishing bilirubin as interference with a single LED light source. However, additional LEDs with different wavelengths can also be integrated to yield a broader spectrum for other applications.

Long-Term Performance. One of the most important features for the hemolysis sensor is the long-term performance. We tested our optical hemolysis sensor rigorously for 7 weeks with a weekly repetition of the test series, while the sensor was integrated as an inline component in the modified ABL90 FLEX blood gas analyzer. A single measurement flow is shown in Figure 5a, where we see the drop of the signal due to the absorption of plasma hemoglobin and its fast recovery after the rinse. The spectra at three different time points are shown in Figure $5 b$, where no drift or remaining absorbency can be observed.

A daily test protocol is shown in Figure 5c, where it starts and terminates with a $450 \mathrm{mg} / \mathrm{L}$ tartrazine dye solution for calibration of the sensitivity. As fresh bovine blood is used for the preparation of the rest of the reagents, fluctuations in $\mathrm{Hb}$ concentration are inevitable and the dye calibration can thus better quantify the long-term change. Hemolyzed whole blood samples with about $100 \mathrm{mg} / \mathrm{dL} \mathrm{Hb}$ concentration (HWB100) are tested with 5 runs, followed by its centrifuged plasma counterparts (plasma100). WB and its plasma counterpart (plasma) are prepared in the same way. To test for interference, bilirubin $(20 \mathrm{mg} / \mathrm{dL})$ is mixed into the plasma sample with 100 $\mathrm{mg} / \mathrm{dL} \mathrm{Hb}$. According to Figure 5c, very little change in sensitivity happens before and after carrying out the measurement series as tartrazine deviates only $0.4 \%$. Very small concentrations of $\mathrm{Hb}$ around $10 \mathrm{mg} / \mathrm{dL}$ in nonhemolyzed samples can be resolved. Only slight deviation is present under high bilirubin concentration.

Observing the whole test period of 47 days, we can find a high daily repeatability and a small drift in the sensitivity, as shown in Figure 5d, where the tartrazine absorption is shown. The results show only a overall drift is $4.7 \%$ after 47 days, which can be attributed to detachment of the nanofilters or slight change in material optical property as the sensor is kept in liquid (even in the standby phase). The results of HWB and the plasma counterparts are plotted in Figure 5e. Variation in sample $\mathrm{Hb}$ concentration is present as the reference $\mathrm{Hb}$ measurements fluctuate between 68 to $115 \mathrm{mg} / \mathrm{dL}$, which helps evaluating the sensor performance upon concentration variations. The pooled standard deviations $\left(S_{\mathrm{p}}\right)$ are 2.6 and $2.3 \mathrm{mg} / \mathrm{dL}$ for whole blood and plasma samples, respectively, showing similar and very good repeatability. From these values, we can calculate the limit of detection to be $4.3 \mathrm{mg} / \mathrm{dL}$ for free hemoglobin sensing in whole blood. Note that the bias to the reference is not considered. The bias between WB and plasma samples is however larger (up to $10 \%$ ). It might originate from the scattered light, which is not guided but still projected onto the camera, and the camera background correction that took place between the HWB and plasma measurements. 
Furthermore, the influence of additional bilirubin in plasma is evaluated. Figure $5 \mathrm{f}$ shows the evaluated $\mathrm{Hb}$ in plasma with bilirubin interference; the $S_{\mathrm{p}}$ of $2.7 \mathrm{mg} / \mathrm{dL}$ is almost the same as that without bilirubin. An overall positive bias with respect to the reference $(7.0 \% \mathrm{CoV}$ in average) can be seen, indicating slightly elevated absorption with the coexistence of bilirubin and $\mathrm{Hb}$. In general, we observed no performance deterioration after 7 weeks, confirming the durability of the hemolysis sensor with the long-term exposure to blood and rinse.

\section{CONCLUSIONS}

In summary, we demonstrated an optofluidic waveguide sensor for in-line hemolysis detection on whole blood with unique, integrated cell/plasma separation ability. The arrangement of nanofilters on top of the waveguide enables local blocking of species out of the evanescent field and allows independent choice of light wavelength and filtration size. The fabrication process can be easily scaled up for industrial production with low cost and high production yield. The sensor can be used as a flexible, standalone device or can be easily integrated into existing PoC platforms. It is shown that a spectrometer-less analysis of spectral extinction of molecules can be successfully achieved by employing out-coupling gratings as a dispersive element and a line camera for readout. We demonstrate here that the portable optical setup is capable of being integrated into a commercial blood gas analyzer. Long-term tests up to 7 weeks were also carried out to demonstrate the high repeatability and reliability of the hemolysis sensor subject to whole blood samples even with the presence of strong interference from bilirubin. We envisage large potential for the sensor to be used inline in blood gas analyzers for hemolysis detection as well as in other fields related to turbid media measurements.

\section{MATERIALS AND METHODS}

Sensor Fabrication and Materials. To fabricate the hemolysis sensor, a borofloat wafer was used as substrate a substrate. The polymer Efiron (Luvantix, South Korea) was drop-cast and imprinted with the silicon master stamp, which is treated with perfluorodecyltrichlorosilane (FTDS). The polymer was cured by UV exposure in an $\mathrm{N}_{2}$ atmosphere, followed by manual separation with a razor blade. The waveguide layer made of OrmoClear $\mathrm{H} 101$ was spin-coated on the Eifron surface treated with oxygen plasma and baked to evaporate solvent. OrmoClear H101 was diluted with propylene glycol methyl ether acetate (PGMEA) to yield the desired thickness. The UV curing of OrmoClear $\mathrm{H} 101$ was done by UV curing in $\mathrm{N}_{2}$ atmosphere. The waveguide stack was oxygen plasma treated before spin-coating with OrmoComp, which was diluted in the same way as OrmoClear H101. After the evaporation of the solvent, the waveguide was aligned with the organic-inorganic hybrid imprint stamp to define the nanofilters. Imprinting was done by applying 6 bar pressure for $20 \mathrm{~min}$ with subsequent UV exposure in a UV-imprinter (CNI v 2.0, NIL Technology, Denmark). A thick layer of OrmoComp can be optionally used to passivate the surface outside of the filter region, which was done with UV-photolithography with a mask aligner (Karl Süss MA6 contact aligner, Germany). Finally, a fluidic channel can be applied by a double-adhesive tape (tesa 62571, Germany) with a cover formed by laser-cut PMMA. Fabrication of the organic-inorganic hybrid imprint stamp started with oxygen plasma treatment of a borofloat glass wafer, followed by spin-coating of an adhesion layer OrmoPrime08. Around $150 \mu \mathrm{L}$ OrmoStamp was suspended on top of the wafer and imprinted with the FTDS-treated master stamp while UV exposure was applied to cure the OrmoStamp, followed by hard-baking at $130{ }^{\circ} \mathrm{C}$ for 15 min. The stamp was finished by treating with FTDS for antisticking property. Here, OrmoPrime08, OrmoStamp, OrmoClear H101,
OrmoComp, PGMEA are acquired from micro resist technology $\mathrm{GmbH}$, Germany.

Optical Simulations. The mode analysis simulations were carried out with COMSOL (COMSOL Multiphysics). Unit cells were defined with Floquet periodicity. Real and imaginary refractive index were acquired by spectroscopic ellipsometry measurements (M-2000, J.A. Woollam) of each material deposited as a thin film onto silicon wafers. OrmoComp $\left(n_{\text {OrmoComp }}=1.537 @ 420 \mathrm{~nm}\right)$, OrmoClear H101 $\left(n_{\text {OrmoClear }}=1.629 @ 420 \mathrm{~nm}\right)$, and Efiron $\left(n_{\text {OrmoComp }}=1.414 @ 420\right.$ $\mathrm{nm}$ ) were modeled for the geometry we obtained. The evanescent overlap was defined by the ratio of power immersed in the liquid to the total power in the waveguide. Unfiltered ratio was defined as power in the area higher than the top of the filter region to the total power in the liquid.

Blood Sample Preparation. Hemolyzed whole blood was prepared by mixing completely hemolyzed fresh whole blood from human or bovine to fresh whole blood, of which the total hemoglobin concentration was measured with a blood gas analyzer in advance (ABL90 FLEX, Radiometer Aps, Denmark). The completely hemolyzed whole blood was obtained by freezing the blood sample in $-20{ }^{\circ} \mathrm{C}$ for at least $3 \mathrm{~h}$. Part of the prepared whole blood sample was centrifuged to obtain the plasma sample. Bilirubin interfered plasma samples were prepared by adding concentrated bilirubin base solution into plasma from the centrifuged whole blood sample containing $100 \mathrm{mg} / \mathrm{dL} \mathrm{Hb}$. The bilirubin base solution was prepared by mixing bilirubin powder (Sigma-Aldrich, USA) into $0.1 \mathrm{M} \mathrm{NaOH}$ solution with a concentration of $10 \mathrm{~g} / \mathrm{L}$. Tartrazine calibration liquid was prepared by mixing tartrzine $(450 \mathrm{mg} / \mathrm{L})$ into the Cal. Two solution from a Radiometer solution pack (REF 944-157, Radiometer Aps, Denmark).

Absorption Characterization. An LED light source (M420L3, Thorlabs) with a peak wavelength of $420 \mathrm{~nm}$ was used for the $\mathrm{Hb}$ absorption test. The light was collimated into a multimode fiber with a core diameter of $200 \mu \mathrm{m}$. The output light was collimated and focused with a plano-convex lens with a focal length of $50 \mathrm{~mm}$. The sensor was placed on a platform with two apertures fitting the size of the grating couplers $(2 \times 2 \mathrm{~mm})$. The out-coupled light was filtered through a linear polarizer and captured by a CMOS linear image sensor (S11639-01, Hamamatsu, Japan). For the calibration of the sensor, 405 and $450 \mathrm{~nm}$ laser diode modules (CPS405, CPS450, Thorlabs) were used to define the spectral locations. During the measurement phosphate buffered saline (PBS) or rinse solution from Radiometer solution pack (REF 944-157, Radiometer ApS, Denmark) were used to rinse the surface of the sensor. Scattering signals on the linear camera were corrected by linear subtraction of signal tails before the absorbance is calculated.

Reference Absorption Measurement. All biological samples were diluted with phosphate buffered saline (PBS) solution according to their concentrations. The following mixing ratios were used for samples in this work: nonhemolyzed plasma 1:3, plasma with $100 \mathrm{mg} /$ $\mathrm{dL} \mathrm{Hb} \mathrm{1:6,} \mathrm{plasma} \mathrm{with} 100 \mathrm{mg} / \mathrm{dL} \mathrm{Hb}$ and $20 \mathrm{mg} / \mathrm{dL}$ bilirubin $1: 12$, tartrazine calibration solution with $450 \mathrm{mg} / \mathrm{L}$ tartrazine: $1: 12$. UVtransparent $1 \mathrm{~cm}$ cuvettes were used for characterization with a UV photospectrometer (UV-1800 Shimadzu Corp., Japan). A spectral range from 330 to $700 \mathrm{~nm}$ was used to characterize the samples for reference.

Long-Term Measurement. During the long-term measurements the optical setup was attached to a modified ABL90 FLEEX blood gas analyzer(Radiometer ApS, Denmark). Two ports were wired through silicone tubing to the in- and outlet of the analyzer. The samples were always acquired through the sample inlet of the analyzer. Each acquisition took around $70 \mu \mathrm{L}$ of the sample, which was delivered to the sensor by the internal peristaltic pump of the analyzer. Five seconds were set to allow the sample in touch with the sensor before the rinse protocol is carried out, which was a modified version of the commercial version of the analyzer, including 5 times air and rinse alternating purge and a final liquid rinse. Recording of the camera was carried out with $2-12 \mathrm{~ms}$ integration time subject to the intensity transmitted. $250 \mathrm{~ms}$ sampling interval was used for real-time recording, which terminated after $20 \mathrm{~s}$ after the rinse protocol. The calculation of 
the different components in the blood was made afterward by nonlinear least-squares fitting with reference spectra. Daily measurement protocol included 5 times repeated HWB 100 and the rest samples were measured 3 times apart from the tartrazine calibration liquid which was measured once before and after all other measurements. Tartrazine $(450 \mathrm{mg} / \mathrm{dL})$ was measured once before and once after the blood and plasma measurements. The evaluation of the long-term sensitivity change was done by observing tartrazine absorption over days, while it was calibrated with the measurements on the first day to $450 \mathrm{mg} / \mathrm{dL}$. Bovine blood was used during the longterm test, which was acquired from Statens Serum Institute, Denmark. After the sample measurements, the analyzer was automated to perform a rinse protocol every $4 \mathrm{~h}$ for circulation and purge.

\section{ASSOCIATED CONTENT}

\section{S Supporting Information}

The Supporting Information is available free of charge on the ACS Publications website at DOI: 10.1021/acssensors. 8 b00030.

System modeling and calculations (PDF)

\section{AUTHOR INFORMATION}

\section{Corresponding Author}

*E-mail: anders.kristensen@nanotech.dtu.dk.

\section{ORCID}

\section{Anders Kristensen: 0000-0001-9567-1656}

\section{Notes}

The authors declare no competing financial interest.

\section{ACKNOWLEDGMENTS}

The research was partially funded by Innovation Fund Denmark through the project HemoPoc (grant number: 572014-3). This project has also received funding from the European Union's Horizon 2020 research and innovation programme under the Marie Sklodowska-Curie grant agreement No 701597.

\section{REFERENCES}

(1) Lippi, G.; Cervellin, G.; Favaloro, E. J.; Plebani, M. In Vitro and In Vivo Hemolysis - An Unresolved Dispute in Laboratory Medicine; DeGruyter: Germany, 2012.

(2) Lippi, G.; Guidi, G. C. Risk management in the preanalytical phase of laboratory testing. Clin. Chem. Lab. Med. 2007, 45, 720-727.

(3) Vercaemst, L. Hemolysis in cardiac surgery patients undergoing cardiopulmonary bypass: a review in search of a treatment algorithm. Journal of Extra-corporeal Technology 2008, 40, 257-67.

(4) Mamikonian, L. S.; Mamo, L. B.; Smith, P. B.; Koo, J.; Lodge, A. J.; Turi, J. L. Cardiopulmonary Bypass is Associated with Hemolysis and Acute Kidney Injury in Neonates, Infants and Children. Pediatr Crit Care Med. 2014, 15, 111-119.

(5) Gupta, R.; Goddard, N. J. Broadband absorption spectroscopy for rapid $\mathrm{pH}$ measurement in small volumes using an integrated porous waveguide. Analyst 2017, 142, 169-176.

(6) Wiederkehr, R. S.; Mendes, S. B. Extension of the broadband single-mode integrated optical waveguide technique to the ultraviolet spectral region and its applications. Analyst 2014, 139, 1396-1402.

(7) Sakota, D.; Takatani, S. Plasma surface reflectance spectroscopy for non-invasive and continuous monitoring of extracellular component of blood. Proc. SPIE 2012, 8439, 1-6.

(8) Bradshaw, J. T.; Mendes, S. B.; Saavedra, S. S. Accelerated Article A Simplified Broadband Coupling Approach Applied to Chemically Robust Sol-Gel, Planar Integrated Optical Waveguides A Simplified Broadband Coupling Approach Applied to Chemically Robust Sol-Gel, Planar Integrated Optical Waveguides. Anal. Chem. 2002, 74, 17511759
(9) Mendes, S. B.; Li, L.; Burke, J. J.; Lee, J. E.; Dunphy, D. R.; Saavedra, S. S. Broad-Band Attenuated Total Reflection Spectroscopy of Waveguide. Langmuir 1996, 12, 3374-3376.

(10) Cadarso, V. J.; Llobera, A.; Puyol, M.; Schift, H. Integrated photonic nanofences: Combining subwavelength waveguides with an enhanced evanescent field for sensing applications. ACS Nano 2016, $10,778-785$.

(11) Ryckeboer, E.; Bockstaele, R.; Vanslembrouck, M.; Baets, R Glucose sensing by waveguide-based absorption spectroscopy on a silicon chip. Biomed. Opt. Express 2014, 5, 1636-1648.

(12) Gupta, R.; Bastani, B.; Goddard, N. J.; Grieve, B. Absorption spectroscopy in microfluidic flow cells using a metal clad leaky waveguide device with a porous gel waveguide layer. Analyst 2013, $138,307-314$.

(13) Zhang, L.; Wang, P.; Xiao, Y.; Yu, H.; Tong, L. Ultra-sensitive microfibre absorption detection in a microfluidic chip. Lab Chip 2011, 11, 3720.

(14) Prabhakar, A.; Mukherji, S. Microfabricated polymer chip with integrated U-bend waveguides for evanescent field absorption based detection. Lab Chip 2010, 10, 748.

(15) Mogensen, K. B.; Friis, P.; Hubner, J.; Petersen, N. J.; Jorgensen, A. M.; Telleman, P.; Kutter, J. P. Ultraviolet transparent silicon oxynitride waveguides for biochemical microsystems. Opt. Lett. 2001, 26, 716-718.

(16) Kim, P.; Ong, E. H.; Li, K. H. H.; Yoon, Y. J.; Ng, S. H. G.; Puttachat, K. Low-cost, disposable microfluidics device for blood plasma extraction using continuously alternating paramagnetic and diamagnetic capture modes. Biomicrofluidics 2016, 10, 024110.

(17) Han, K.-H.; Frazier, A. B. Paramagnetic capture mode magnetophoretic microseparator for high efficiency blood cell separations. Lab Chip 2006, 6, 265-273.

(18) Nakashima, Y.; Hata, S.; Yasuda, T. Blood plasma separation and extraction from a minute amount of blood using dielectrophoretic and capillary forces. Sens. Actuators, B 2010, 145, 561-569.

(19) Jung; Kwak, H.-Y. Separation of Microparticles and Biological Cells Inside an Evaporating Droplet Using Dielectrophoresis. Anal. Chem. 2007, 79, 5087-5092.

(20) Lenshof, A.; Ahmad-Tajudin, A.; Järås, K.; Swärd-Nilsson, A.M.; Åberg, L.; Marko-Varga, G.; Malm, J.; Lilja, H.; Laurell, T. Acoustic Whole Blood Plasmapheresis Chip for Prostate Specific Antigen Microarray Diagnostics. Anal. Chem. 2009, 81, 6030-6037.

(21) Petersson, F.; Nilsson, A.; Holm, C.; Jönsson, H.; Laurell, T. Separation of lipids from blood utilizing ultrasonic standing waves in microfluidic channels. Analyst 2004, 129, 938-943.

(22) Prabhakar, A.; Kumar, Y. V. B. V.; Tripathi, S.; Agrawal, A. A novel, compact and efficient microchannel arrangement with multiple hydrodynamic effects for blood plasma separation. Microfluid. Nanofluid. 2015, 18, 995-1006.

(23) Kersaudy-Kerhoas, M.; Kavanagh, D. M.; Dhariwal, R. S.; Campbell, C. J.; Desmulliez, M. P. Y. Validation of a blood plasma separation system by biomarker detection. Lab Chip 2010, 10, 1587.

(24) Mach, A. J.; di Carlo, D. Continuous scalable blood filtration device using inertial microfluidics. Biotechnol. Bioeng. 2010, 107, 302311.

(25) Zhang, X. B.; Wu, Z. Q.; Wang, K.; Zhu, J.; Xu, J. J.; Xia, X. H.; Chen, H. Y. Gravitational sedimentation induced blood delamination for continuous plasma separation on a microfluidics chip. Anal. Chem. 2012, 84, 3780-3786.

(26) Sun, M.; Khan, Z. S.; Vanapalli, S. a. Blood plasma separation in a long two-phase plug flowing through disposable tubing. Lab Chip 2012, 12, 5225-5230.

(27) Aran, K.; Fok, A.; Sasso, L. A.; Kamdar, N.; Guan, Y.; Sun, Q.; Ündar, A.; Zahn, J. D. Microfiltration platform for continuous blood plasma protein extraction from whole blood during cardiac surgery. Lab Chip 2011, 11, 2858.

(28) Thorslund, S.; Klett, O.; Nikolajeff, F.; Markides, K.; Bergquist, J. A hybrid poly(dimethylsiloxane) microsystem for on-chip whole blood filtration optimized for steroid screening. Biomed. Microdevices 2006, 8, 73-79. 
(29) Kang, T. G.; Yoon, Y.-J.; Ji, H.; Lim, P. Y.; Chen, Y. A continuous flow micro filtration device for plasma/blood separation using submicron vertical pillar gap structures. J. Micromech. Microeng. 2014, 24, 087001.

(30) Shim, J. S.; Ahn, C. H. An on-chip whole blood/plasma separator using hetero-packed beads at the inlet of a microchannel. Lab Chip 2012, 12, 863.

(31) Mortensen, N. A.; Xiao, S.; Pedersen, J. Liquid-infiltrated photonic crystals: enhanced light-matter interactions for lab-on-a-chip applications. Microfluid. Nanofluid. 2008, 4, 117-127.

(32) Bhushan, B. E., Ed. Springer Handbook of Nanotechnology, 3rd ed.; Springer: Berlin Heidelberg, 2010; Chapter 9, pp 271-312.

(33) Mauranyapin, N. P.; Madsen, L. S.; Taylor, M. A.; Waleed, M.; Bowen, W. P. Evanescent single-molecule biosensing with quantumlimited precision. Nat. Photonics 2017, 1-6.

(34) Shao, L.; Jiang, X.-F.; Yu, X.-C.; Li, B.-B.; Clements, W. R.; Vollmer, F.; Wang, W.; Xiao, Y.-F.; Gong, Q. Detection of Single Nanoparticles and Lentiviruses Using Microcavity Resonance Broadening. Adv. Mater. 2013, 25, 5616-5620.

(35) Zhu, J.; Ozdemir, S. K.; Xiao, Y.-F.; Li, L.; He, L.; Chen, D.-R.; Yang, L. On-chip single nanoparticle detection and sizing by mode splitting in an ultrahigh-Q microresonator. Nat. Photonics 2010, 4, 4649.

(36) He, L.; Özdemir, a. K.; Zhu, J.; Kim, W.; Yang, L. Detecting single viruses and nanoparticles using whispering gallery microlasers. Nat. Nanotechnol. 2011, 6, 428-432.

(37) Baaske, M. D.; Foreman, M. R.; Vollmer, F. Single-molecule nucleic acid interactions monitored on a label-free microcavity biosensor platform. Nat. Nanotechnol. 2014, 9, 933-9.

(38) Baaske, M. D.; Vollmer, F. Optical observation of single atomic ions interacting with plasmonic nanorods in aqueous solution. Nat. Photonics 2016, 10, 733-739.

(39) Koseoglu, M.; Hur, A.; Atay, A.; Cuhadar, S. Effects of hemolysis interference on routine biochemistry parameters. Biochemia Medica 2011, 79-85.

(40) U.S. Department of Health and Human Services, Public Health Service, Centers for Disease Control, P. H. P. O. CLIA '88 Focus on Clinic and Office Laboratories, 1988.

(41) EDQM, Guide to the preparation, use and quality assurance of blood components, Recommendation No. R (95) 15.

(42) Provan, D. Medicine; 2009; Vol. 98; Chapter 12, p 920.

(43) Roca, L.; Calligaris, S.; Wennberg, R. P.; Ahlfors, C. E.; Malik, S. G.; Ostrow, J. D.; Tiribelli, C. Factors affecting the binding of bilirubin to serum albumins: Validation and application of the peroxidase method. Pediatr. Res. 2006, 60, 724-728.

(44) Ardakani, S. B.; Dana, V. G.; Ziaee, V.; Ashtiani, M.-T. H.; Djavid, G. E.; Alijani, M. Bilirubin/Albumin Ratio for Predicting Acute Bilirubin-induced Neurologic Dysfunction. Iranian Journal of Pediatrics 2011, 21, 28-32. 